\title{
REZENSIONEN
}

\section{Jahre Grundgesetz: Anleitungen zu Respekt, Kritik und Verteidigung}

Möllers, Christoph: Das Grundgesetz. Geschichte und Inhalt, Verlag C. H. Beck, München 2009, 122 Seiten, $€ 8,95$.

Bommarius, Christian: Das Grundgesetz. Eine Biographie, 2. Auflage, Rowohlt Berlin Verlag, Berlin 2009, € 19,90.

Auf der Website der Bundeszentrale für politische Bildung sind einige eindrückliche Fotos zur Entstehungsphase des Grundgesetzes zu finden. Eines der Bilder zeigt eine Handvoll Neugieriger, die von außen durch die Fensterscheibe die konstituierende Sitzung des Parlamentarischen Rates am 1. September 1948 im Bonner Museum Alexander Koenig verfolgen, in einem Lichthof, der eigentlich als Ausstellungsfläche für Tierpräparate diente. Die Besuchertribüne ist zwar voll besetzt, doch fasst sie nicht viele Zuschauer. Bereits im September 1948 beklagte der liberale Politiker Thomas Dehler die „fehlende Resonanz, in unserem müde und skeptisch gewordenen deutschen Volke“" (Bommarius, S. 10). So leidenschaftlich das Grundgesetz erst von den Mitgliedern des Verfassungskonvents auf Herrenchiemsee, dann vom Parlamentarischen Rat in Bonn diskutiert wurde, so teilnahmslos waren die Westdeutschen. Neben Hunger und Not spielte die verbreitete Annahme eine Rolle, dass das Grundgesetz „Satz für Satz von den Siegermächten USA, Großbritannien und Frankreich diktiert und genehmigt" (S. 11) worden war und sich ein Einsatz daher gar nicht lohne. Eindrücklich belegt Christian Bommarius in seiner „Biographie“ des Grundgesetzes, wie falsch diese Vermutung ist.

Was ist eine „Biographie“ des Grundgesetzes? Bommarius füllt diesen Titel aus, indem er aufzeigt, welche Rolle Personen und die in ihren Lebenserfahrungen und Überzeugungen begründeten Positionen für die Konzeption des Grundgesetzes in seiner ursprünglichen Version gespielt haben. Die Biographie der Verfassung ist die Lebensgeschichte eines kollektiven Akteurs, der vorwiegend im Widerstand und der Emigration überlebte oder zumindest in das Naziregime nicht verstrickt war, und gerade deshalb in der Lage war, den Grundstein für ein demokratisches Deutschland zu legen. Für Bommarius wie auch für Christoph Möllers steht außer Frage, dass der Parlamentarische Rat das deutsche Volk nicht repräsentierte und damit bis zur Gründung des Bundesverfassungsgerichts das „einzige relevante staatliche Organ in Westdeutschland [blieb], in dem die politische Opposition zum Nationalsozialismus eine Mehrheit hatte und damit stimmungs- und meinungsbildend wirken konnte" (Möllers, S. 23 f.). Ohne die Besatzungsmächte hätte diese Minderheit kaum zum Verfassunggeber werden können. Beiden Umständen ist zu verdanken, dass im Grundgesetz eine beeindruckende „kopernikanische Wende“ (Bommarius, S. 114) im Staatsverständnis vollzogen wurde: Der Staat dient den Menschen, und die Würde des Menschen ist unantastbar.

Bommarius verfolgt in seinem Buch zunächst die verästelte Entstehungsgeschichte dieser Grundnormen des Grundgesetzes. Faszinierend sind die Wege der Kernüberzeugungen zu 
verfolgen, die Eingang in den Verfassungstext gefunden haben. Das Bekenntnis zur Menschenwürde führt Bommarius auf die „Grundsätze für die Neuordnung“ Deutschlands nach dem Krieg zurück, die der Kreisauer Kreis 1943 formuliert hatte. Dessen Programm enthielt „fast alle Leitgedanken, die die Verfassungsdebatten des deutschen Widerstands ebenso wie der verstreuten Emigration und später in Westdeutschland beherrschten" (S. 49), darunter die unverletzliche Würde der Person als wichtigste Basis einer neuen Rechtsund Friedensordnung. Als Urheber des Begriffs „Grundgesetz“ identifiziert er den Ersten Hamburger Bürgermeister, Max Brauer (S. 140). Der Wirtschaftsjournalist, Sozialdemokrat und Gewerkschafter Fritz (Perez) Naphtali, der durch den beherzten Einsatz seines Parteifreundes Hans Staudinger dem Konzentrationslager entging, lieferte die Grundsätze der Wirtschaftsdemokratie. Naphtali emigrierte nach Israel, seine Überlegungen zur planmäßigen Wirtschaftsgestaltung blieben nicht nur in der Sozialdemokratie erhalten, sondern wurden in allen fast allen Landesverfassungen aufgegriffen (vgl. S. 86 ff.). Hier wird auch der Grundstein für die Wende gelegt: Das „Selbstverständnis der neuen Republik [...] entsteht auf den Trümmern des zerstörten Bildes, in dem der deutsche Staat sich jahrhundertelang zu erkennen glaubte. Die Westdeutschen haben es nach dem Krieg nicht zusammengesetzt. Sie haben ein neues gemalt, nicht erst im Parlamentarischen Rat, sondern in den Verfassunggebenden Versammlungen der Länder. Es waren damals viele Maler am Werk, Vertreter der verschiedenen Schulen, aber die Staats-Bilder, die damals entstanden, glichen einander in den wesentlichen Punkten bis ins Detail. Das Bild, in dem der neue Staat sich künftig selbst erkennen sollte, zeigte ihn nicht mehr als Herrn, sondern als Diener der Menschen, nicht mehr allmächtig, sondern an die Grundrechte gebunden. Als sich der Parlamentarische Rat darüber beugte, war das Bild fast fertig. " (S. 114)

Dieser Austausch der Grundüberzeugung, so Bommarius, blieb fast unbemerkt, wurde gleichsam geräuschlos vollzogen, getragen von der Einmütigkeit der Verfassunggeber in Ländern und Bund und übertönt vom Gerangel um institutionelle Fragen, die die Konfliktlinien der Arbeit am Verfassungstext bestimmten: Die Stellung der Länder zum Bund, Bundesrat oder Senat, Steuererhebung und Finanzverfassung und, nicht zuletzt, das Notverordnungsrecht (S. 145). Welche Antworten auf diese Fragen gefunden wurden und wie auch die weiteren Grundrechte, vor allem die Gleichberechtigung von Mann und Frau, das Asylrecht und das Kriegsdienstverweigerungsrecht, in den Debatten des Parlamentarischen Rates ausgestaltet wurden, weist Bommarius kenntnisreich und spannend nach (S. $274 \mathrm{ff}$.). Dass überhaupt Antworten gefunden werden konnten, kann durch den Grundkonsens der Verfassunggeber erklärt werden. Dass das Grundgesetz im Verlauf der Bundesrepublik aber Bestand hatte, kann mit der Güte des Gesetzes selbst nicht hinreichend erklärt werden. Verfassungsfragen sind Machtfragen, und in der Entstehung des Grundgesetzes war eine Minderheit am Werk, die auf keinen Rückhalt der Bevölkerung hoffen konnte. „Eine Verfassung ist nur so stark wie der Glaube der Bürger an die Grundsätze, die sie formuliert. Kraftloser als das Grundgesetz [...] hat kaum je eine demokratische Verfassung begonnen. Das Gefühl, das sie bei den Westdeutschen hervorrief, war weder Begeisterung noch Ablehnung, sondern Teilnahmslosigkeit.“ (S. 10) Der Erfolg des Grundgesetzes und seine Stärke, so die These, verdankt sich nicht seinen Bürgern, sondern dem Bundesverfassungsgericht. „Demokratie als offenes Gespräch - was das sein, wie das funktionieren kann, haben die Westdeutschen weniger im Parteienstreit gelernt und in den Parlamentsdebatten, sondern im Gericht, genauer: durch das Bundesverfassungsgericht. Es hat das Grundgesetz zum Sprechen gebracht." (S. 218) 
Christian Bommarius widmet sein Buch den Mitgliedern des Parlamentarischen Rates. Diese Widmung überzeugt, denn der Respekt vor ihrer Leistung bestimmt seine engagierte Rekonstruktion der Erfolgsgeschichte des Grundgesetzes. Als Motiv des Buches tritt neben Achtung aber auch eine nicht weniger leidenschaftliche Sorge vor einer weiteren Erosion der Werte des Grundgesetzes. Im abschließenden Kapitel zeichnet Bommarius die „Chronik einer Belagerung" (S. 255), nämlich die zunehmenden Angriffe auf den Wesensgehalt von Artikel 1, der unantastbaren Menschenwürde. Scharf und überzeugend kritisiert er die Rechtsentwicklung des letzten Jahrzehnts: Rasterfahndung, Luftsicherheitsgesetz, Präventionsstaat, Aushöhlung des Asylrechts bis zur Unkenntlichkeit - all dies sind Zeichen, dass „nicht nur der deutsche Gesetzgeber [versucht], die ,kopernikanische Wende‘ im Staatsverständnis und im Menschenbild zurückzunehmen“ (S. 257). Den Deutschen ist das Grundgesetz geschenkt worden, so kann man die Auffassung von Christian Bommarius resümieren, jetzt sind die Bürger in der Pflicht, ihr Grundgesetz zu verteidigen. „Die Deutschen - zumindest die Westdeutschen - haben sich die Freiheit nicht erkämpft, sie wurde ihnen mit dem Grundgesetz in die Wiege gelegt. Vielleicht können sie deshalb nicht recht erkennen, wann es Zeit ist, sie zu verteidigen." (S. 254) Viele Hoffnungen hegt Bommarius nicht: „Groß ist zwar nach 60 Jahren die Liebe der Deutschen zum Grundgesetz, aber offenbar nicht groß genug, ihm und seinem Hüter in schwerster Bedrängnis zu Hilfe zu eilen." (S. 263)

Christoph Möllers' Buch zu Geschichte und Inhalt des Grundgesetzes entstammt einem anderen Genre der Verfassungsliteratur. Als Einführung konzipiert erläutert es knapp und strukturiert die Vorgeschichte und Entstehung (Kapitel 1), das Grundgesetz als Text (Kapitel 2), als Norm (Kapitel 3), als Kultur (Kapitel 4) und schließt mit einem Ausblick auf die Herausforderungen, vor denen das Grundgesetz steht (Kapitel 5). Gerade weil es sich um eine andere Textsorte handelt und weil jeder der Bände für sich klug konzipiert ist, sind beide Bücher mit großem Gewinn nacheinander zu lesen: Möllers verschafft ein grundlegendes, gleichwohl pointiertes Verständnis der Rechtsordnung und des Grundgesetzes, Bommarius zeigt, woher die Überzeugungen rühren, von denen beide getragen werden, und wirbt für deren Fortbestand.

Wie gezeigt macht auch Möllers deutlich, dass die Legitimation des Verfassunggebers sich nicht auf den Repräsentationsgedanken stützen kann. Dieser vielfach beklagte „,Geburtsmakel““ (Möllers, S. 37) ist aber seiner Auffassung nach für Verfassungen weder untypisch noch von Belang. Die Legitimation des Grundgesetzes „folgte aus der Praxis demokratischer Selbstbestimmung, vor allem in den ersten Bundestagswahlen des Jahres 1949, die durch das Inkrafttreten des Grundgesetzes erst ermöglicht wurden“ (S. 38). Mit der Wiedervereinigung stellte sich diese Frage der Legitimation erneut. Deutlich wurde dies in der Forderung nach der Anwendung von Art. 146 GG, der eine demokratische Neugründung durch den gemeinsamen Akt einer verfassunggebenden Gewalt des Volkes ermöglicht hätte. Dass die Wiedervereinigung stattdessen nach Art. 23 GG (alte Fassung) vollzogen wurde, als Beitritt der neuen Länder, führte bekanntlich zur Beibehaltung des Grundgesetzes. Möllers beurteilt diese Entscheidung nüchtern: Erstens habe kein Anlass bestanden, einen gänzlich neuen Text zu entwerfen. Zweitens aber werde in der Beibehaltung des Grundgesetzes nur deutlich, dass das Bedürfnis nach einer gemeinsamen politischen Ordnung in Ost und West nicht sehr ausgeprägt war. „Eine gesamtdeutsche Verfassunggebung - und sei es nur als neues Inkrafttreten des Grundgesetzes - hätte die symbolisch-politische Gemeinsamkeit an den Anfang des Einigungsprozesses gestellt, mit der wir uns bis heute so 
schwer tun. [...] Verfassungen bestimmen nicht, wie eine demokratische Ordnung ist, sondern welchen normativen Anspruch sie an sich selbst erhebt, also wie sie sein will. Die Wiedervereinigung als Vertrag zu vollziehen, zeigt, wie schwach der Anspruch der Beteiligten war, eine wirkliche gemeinsame Ordnung zu vollziehen." (S. 83 f.)

Deutliche Position bezieht Möllers auch in der Frage der Textveränderungen des Grundgesetzes und dem damit teilweise einhergegangen Sprachwandel. Ursprünglich in einer „schmucklosen, aber schönen Sprache“ (S. 59) verfasst, haben auch im internationalen Vergleich zahlreiche Änderungen zu einer wortreichen Ausdehnung des schlichten Textes geführt. „Sie verderben den Leseeindruck, und sie nehmen dem Text viel von der politischen Würde, die sich aus der einfachen und klaren Definition wichtiger Rechtsätze, nicht aus der technisch unbeholfenen Formulierung von Details ergibt." (S. 61)

Auch für Möllers ist die Bedeutung des Bundesverfassungsgerichts für die Durchsetzung der Rechtsordnung in der Bundesrepublik fraglos. Der Verfassungsvorrang, der in Art. 20 Abs. 3 GG kodifiziert ist, bedurfte zweier Instrumente, um auch institutionell wirksam zu werden. Hierfür „müssen erstens Änderungen der Verfassung gegenüber Änderungen normaler Gesetze in einem erschwerten Verfahren vorgenommen werden. [...] Zum anderen bedarf es einer Institution, die die Einhaltung des Grundgesetzes kontrolliert - und die dabei vom politischen Prozess, der überprüft werden soll, unabhängig ist, also eines Verfassungsgerichts" (S. 62). Die Einrichtung des Bundesverfassungsgerichts ist für Möllers eine „typisch posttotalitäre Entscheidung“ (S. 64), nämlich ein Versuch, das Versagen der Politik durch Recht zu korrigieren. Von dieser Möglichkeit haben die Karlsruher Richter ausgiebig Gebrauch gemacht, was unter anderem zu einer weitgehenden Identifikation des Grundgesetzes mit dem Bundesverfassungsgericht geführt hat. Möllers spricht sich hier jedoch für eine Relativierung aus: Erstens ist eine starke Verfassungsgerichtsbarkeit keine Selbstverständlichkeit, zweitens kann das Gericht die „politischen Kontexte, in denen sie steht, nicht einfach ignorieren“ (S. 65), drittens ist das Gericht in „vielfacher Hinsicht auf außerrechtliche Umstände und auf die anderen Verfassungsorgane verwiesen“ (ebenda). Alles in allem ist das Bundesverfassungsgericht damit „,bei weitem nicht der einzige Akteur, den wir betrachten müssen, um die Entwicklung des Grundgesetzes beschreiben und verstehen zu können“" (S. 66). Stärker als Bommarius beleuchtet Möllers daher die außerrechtlichen institutionellen und politischen Umgebungsvariablen des Grundgesetzes. Nur zuzustimmen ist ihm, dass auch der Bundestag, die Bundesregierung und der Bundespräsident für das Verständnis des Grundgesetzes wichtig sind, und ohne Zweifel müssen öffentliche Sicherheit und politischer Extremismus, Religion, demokratische Öffentlichkeit, soziale Gerechtigkeit, Europa und die internationale Ordnung (S. 100 ff.) als zentrale politische Herausforderungen der Zukunft gesehen werden, in denen sich das Grundgesetz bewähren muss.

Das gemeinsame Fazit ist eine starke Lektüreempfehlung für beide Bücher: Recht gilt zu Unrecht als trockene Materie, und das Grundgesetz verdient nicht nur Respekt, es muss auch verteidigt werden, um weiterhin Bestand zu haben. 